\title{
ON THE HOMOLOGY OF FIBER SPACES
}

\author{
MICHAEL GINSBURG ${ }^{1}$
}

Let $(F, T, X, \pi)$ be a fiber space, with fiber $F$, base $X$, total space $T$, and fiber map $\pi$. A general problem of great interest is that of computing the homology groups of one of the spaces involved, usually $F$ or $T$, in terms of the homology groups of the other two spaces and, perhaps, some other invariants of the fiber space. In this paper we show how the Lusternik-Schnirelmann category of $X$ enters into this problem and affects the relations which may exist between the homology groups of $F, T$, and $X$.

Our main results are stated as theorems and corollaries in $\$ \S 3$ and 4 of this paper, and are summarized here. Let $\Omega X$ denote the space of loops on $X$. If $\operatorname{cat}(X) \leqq k$, we obtain a spectral sequence, $\dot{E}^{r}$, which relates $H(F)$ and $H(\Omega X)$ with $H(T)$ and for which the differentials, $d^{r}$, and groups, $\dot{E}_{p}^{r}$, vanish if $r, p \geqq k$. If cat $(X) \leqq 2$, we obtain an infinite exact sequence relating $H(X), H(F)$, and $H(T)$ which generalizes the Wang sequence. This allows us to compute the additive structure of $H(\Omega X)$ and to partially determine the Pontryagin ring $H_{*}(\Omega X)$. We also consider the Leray-Serre spectral sequence of the fiber space and essentially compute all the differentials if cat $(X) \leqq 2$.

Our method is to replace the chain group of $T$ by a twisted tensor product, $\bar{B} A \tilde{\otimes} C(F)$, where $C(Y)$ denotes the group of chains of $Y, A=C(\Omega X)$, and $\bar{B} A$ is the "bar construction" on $A$. We then apply certain results of $[5]$ which relate $\operatorname{cat}(X)$ and $\bar{B} A$. The necessary definitions and preliminary material are covered in $\$ \S 1$ and 2 , while the proofs of the main theorems are in $\$ 5$.

Some related results are contained in [6]. In that paper we also obtain some results involving the "category of a map," similar to those obtained here by using the "category of a space."

1. Fiber spaces. Let $X$ be a space with base point $x_{0}$. Let $P X$ denote the space of Moore paths on $X$ (see [3]). Thus if $R^{+}$denotes the non-negative real numbers and $I_{r}=[0, r]$ for $r \in R^{+}$, then

$$
P X=\left\{\alpha_{r} \mid \alpha_{r}: I_{r} \rightarrow X, r \in R^{+}\right\} .
$$

A product, $\alpha_{r} \cdot \beta_{s}$, is defined in $P X$ if $\alpha_{r}(r)=\beta_{s}(0)$. If we let $E X$ $=\left\{\alpha_{r} \in P X \mid \alpha_{r}(r)=x_{0}\right\}$ and $p: E X \rightarrow X$ be given by $p\left(\alpha_{r}\right)=\alpha_{r}(0)$,

Received by the editors February 12, 1963.

1 This research was partially supported by the Air Force Office of Scientific Research contract number AF 49(638)-568. 
then $p$ is a map. $\Omega X=p^{-1}\left(x_{0}\right)$, called the loop-space of $X$, is an associative $H$-space.

For convenience of notation we will identify the constant paths of $P X$ with the points of $X$. Thus, the path $\alpha_{0}: I_{0} \rightarrow x$ will be denoted simply by $x$. In this way, $x_{0}$ becomes the unit for the multiplication in $P X$.

Let $\pi: T \rightarrow X$ be a map and let

$$
U_{\pi}=\left\{\left(\alpha_{r}, t\right) \in P X \times T \mid \alpha_{r}(r)=\pi(t)\right\} .
$$

A lifting function for $\pi$ is a map $\lambda: U_{\pi} \rightarrow T$ such that

1. $\pi \lambda\left(\alpha_{r}, t\right)=\alpha_{r}(0)$

2. $\lambda(x, t)=t$ for all $x \in X$.

The lifting function $\lambda$ is called weakly transitive if

$$
\lambda\left(\alpha_{r} \cdot \beta_{s}, t\right)=\lambda\left(\alpha_{r}, \lambda\left(\beta_{s}, t\right)\right)
$$

whenever $\alpha_{r}(r)=\beta_{s}(0)=x_{0}$ and $\left(\beta_{s}, t\right) \in U_{\pi}$. We will call $(F, T, X, \pi, \lambda)$ a (weakly transitive) fiber space if $\lambda$ is a (weakly transitive) lifting function for the map $\pi: T \rightarrow X . F=\pi^{-1}\left(x_{0}\right)$ is called the fiber.

It is easy to show that our definition of fiber space is equivalent to requiring that $\pi$ have the strong covering homotopy property for all spaces. Furthermore, every fiber space is fiber-homotopically equivalent to a weakly-transitive one, as noted by Brown [2]. An example of a fiber space which actually admits a weakly transitive lifting function is $(\Omega X, E X, X, p)$.

Let $A$ be a $D G A$ algebra over the ring $Z$ of integers, and let $\bar{B} A$ denote the bar construction on $A$ (see [3] or [4]). The notation involved in the definition of $\bar{B} A$ is

$$
\begin{aligned}
\bar{A} & =A / Z, \\
\bar{A}^{0} & =Z, \\
\bar{A}^{k} & =\bar{A} \otimes \cdots \otimes \bar{A}, k \text { times, } k \geqq 1, \\
\bar{B} A & =\sum_{k=0}^{k=\infty} \bar{A}^{k}, \text { the direct sum. }
\end{aligned}
$$

As usual, we write $\left[a_{1}, \cdots, a_{k}\right]$ for $a_{1} \otimes \cdots \otimes a_{k} \in \bar{A}^{k}$ and [ ] for the unit of $\bar{A}^{0} . \bar{B} A$ is a chain complex with differential $\bar{d}$ and a gradation given by

$$
\text { degree }\left[a_{1}, \cdots, a_{k}\right]=k+\sum_{i=1}^{i=k} \operatorname{degree} a_{i}
$$

for $a_{i}$ homogeneous elements of $\bar{A}$. Let $\bar{B}_{n} A$ denote the elements of $\bar{B} A$ of degree $n$, and let $\bar{B}^{n} A=\sum_{k=0}^{k=n} \bar{A}^{k}$. 
Let $C(Y)$ denote the group of normalized singular chains of the space $Y$. We will adopt the convention that if $Y$ is arcwise connected, $C(Y)$ will mean chains whose vertices are all at the base point of $Y$. Now let $(F, T, X, \pi, \lambda)$ be a weakly-transitive fiber space. $A=C(\Omega X)$ is a $D G A$ algebra [3], while by restriction, $\lambda$ induces a map $\Omega X \times F \rightarrow F$ which in the usual way induces a map $\bar{\lambda}: A \otimes C(F) \rightarrow C(F) . \bar{\lambda}$ thus defines an $A$-module structure on $C(F)$, and we will write $\bar{\lambda}(a \otimes b)$ $=a \cdot b$. Let $\bar{B} A \tilde{\otimes} C(F)$ denote the usual tensor product of graded groups, with the twisted differential $\tilde{d}$ defined by

$$
\begin{aligned}
\tilde{d}\left(\left[a_{1}, \cdots, a_{k}\right] \otimes c\right)=\bar{d} & {\left[a_{1}, \cdots, a_{k}\right] \otimes c+(-1)^{p}\left[a_{1}, \cdots, a_{k}\right] \otimes \partial c } \\
& +(-1)^{p+\text { degree } a_{k}}\left[a_{1}, \cdots, a_{k-1}\right] \otimes a_{k} \cdot c
\end{aligned}
$$

for $a_{i}$ homogeneous elements of $\bar{A}, c \in C(F)$, and degree $\left[a_{1}, \cdots, a_{k}\right]$ $=p$. It is shown in [2] that $\bar{B} A \tilde{\otimes} C(F)$ is a chain complex; the subcomplex $Z \tilde{\otimes} C(F)$ is isomorphic, as a chain complex, to $C(F)$ and will be denoted simply $C(F)$.

Let $\widetilde{D}_{n}=\sum_{\substack{k=0 \\ k=n}} \bar{B}_{k} A \tilde{\otimes} C(F)$. Then $\widetilde{D}_{0}=C(F), \widetilde{D}_{n} \subseteq \widetilde{D}_{n+1}$, and the subcomplexes $\tilde{D}_{i}$ form a filtration of $\bar{B} A \tilde{\otimes} C(F)$. This gives rise, in the usual way, to a spectral sequence $\tilde{E}^{r}$.

THEOREM 1.1. If $(F, T, X, \pi, \lambda)$ is a weakly transitive fiber space and $\pi_{1}(X)=0$, then

(i) the exact homology sequence of the pair $(T, F)$ is isomorphic to that of the pair $(\bar{B} A \tilde{\otimes} C(F), C(F))$;

(ii) for $r \geqq 2$, the spectral sequence $\tilde{E}^{r}$ is isomorphic to the LeraySerre spectral sequence (see [8]) of the fiber map $\pi$.

This proposition is merely a résumé of statements in [2].

2. Category. Let $X^{k}$ denote the $k$-fold cartesian product of $X$, and let

$$
T^{k}(X)=\left\{\left(x_{1}, \cdots, x_{k}\right) \in X^{k} \mid x_{i}=x_{0} \text { for some } 1 \leqq i \leqq k\right\} .
$$

Choose $x_{0}^{k}$ as the base point of both $X^{k}$ and $T^{k}(X)$. Then by definition $\operatorname{cat}(X) \leqq k$ if and only if the diagonal map of $X$ into $X^{k}$ can be deformed, preserving the base points, into $T^{k}(X)$. This is equivalent to the classical definition if $X$ is separable, metric, an ANR, and $x_{0}$ is a nondegenerate base point in the sense of Puppe [7]. (Classically, $\operatorname{cat}(X) \leqq k$ if $X$ can be covered by $k$ open (or closed) sets each of which is contractible to a point in $X$.)

Again let $A=C(\Omega X)$, and let $j: \bar{B}^{n} A \rightarrow \bar{B} A$ be the inclusion map.

THEOREM 2.1. If $\pi_{1}(X)=0$ and $\operatorname{cat}(X) \leqq k$, there exists a chain map 
$\theta: \bar{B} A \rightarrow \bar{B}^{k-1} A$ such that $j_{*} \theta_{*}: H(\bar{B} A) \rightarrow H(\bar{B} A)$ is the identity isomorphism.

Proof. The author has constructed spaces $W$ and $W_{k-1}, W_{k-1} \subseteq W$, and maps $\phi, \psi, p$, and $f$ such that the diagram

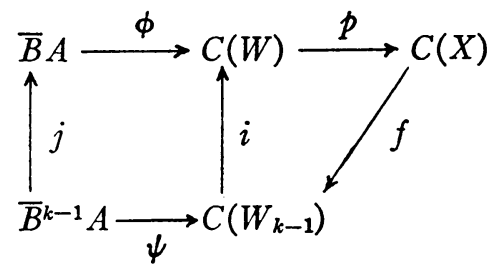

is commutative, where $i$ is induced by inclusion. Furthermore $\phi, \psi$, and $p$ are chain equivalences (see [5, Theorem 3.3 and the proof of Theorem 2.2]). Let $\psi^{-1}$ be any chain inverse to $\psi$, and define $\theta=\psi^{-1} f p \phi . \theta$ is then a chain map. Since $\left(\psi^{-1}\right)_{*}$ is really the isomorphism inverse to $\psi_{*}$, and $i_{*} f_{*}=p_{*}^{-1}$, we have

$$
j_{*} \theta_{*}=j_{*} \psi_{*}^{-1} f_{*} p_{*} \phi_{*}=\phi_{*}^{-1} i_{*} f_{*} p_{*} \phi_{*}=\phi_{*}^{-1} p_{*}^{-1} p_{*} \phi_{*}=\mathrm{id} .
$$

It follows from Theorem 2.1 that if $\operatorname{cat}(X) \leqq 2$ and $(F, T, X, \pi, \lambda)$ is a weakly transitive fiber space, we can define a map $\lambda_{*}: H_{p}(X \times F)$ $\rightarrow H_{p-1}(F)$ as follows. Let $A=C(\Omega X)$ be written as the direct sum $\bar{A}+Z$. Then the map $S: \bar{B}^{1} A \rightarrow A$, defined by $S[a]=a$ and $S[]=0$, lowers the degree by 1 unit and anti-commutes with the boundary operators (see [3]). It is simple to check that $S \theta \otimes 1: \bar{B} A \otimes C(F)$ $\rightarrow A \otimes C(F)$ has the same property. Then the map $\bar{\lambda}(S \theta \otimes 1): \bar{B} A \otimes C(F)$ $\rightarrow C(F)$ induces the desired map $\lambda_{*}$ on homology groups. In the next section we will study this map $\lambda_{*}$.

3. The case $\operatorname{cat}(X) \leqq 2$. In this section it will always be assumed that $(F, T, X, \pi, \lambda)$ is a weakly transitive fiber space with $\pi_{1}(X)=0$ and $\operatorname{cat}(X) \leqq 2$. All homology groups considered are supposed to have coefficients in a fixed principal ideal domain, which will not be explicitly displayed in the notation. Let $i_{*}: H_{n}(X \times F) \rightarrow H_{n}\left(X \times F, x_{0} \times F\right)$ be the epimorphism induced by inclusion.

THEOREM 3.1. There exists an infinite exact sequence

$$
\cdots \rightarrow H_{n}(F) \stackrel{f}{\rightarrow} H_{n}(T) \rightarrow H_{n}\left(X \times F, x_{0} \times F\right) \stackrel{g}{\rightarrow} H_{n-1}(F) \rightarrow \cdots,
$$

where $f$ is induced by inclusion and $g=-\lambda_{*} i_{*}^{-1}$.

Let $H(X) \otimes H(F)$ be graded in the usual way, and identify $H(F)$ with $H_{0}(X) \otimes H(F) . H(X) \otimes H(F)$ is a natural subgroup of $H(X \times F)$; 
let $\lambda_{*}^{\prime}=\lambda_{*} \mid H(X) \otimes H(F)$. Clearly $\lambda_{*}^{\prime} \lambda_{*}^{\prime}=0$ and $\lambda_{*}^{\prime}$ lowers degree by one unit, so we may consider the homology groups of $H(X) \otimes H(F)$ with this differential. The following corollary follows immediately from Theorem 3.1.

Corollary 1. Suppose $H(X)$ and $H(F)$ are both free. Then $H(T)$ $\approx H(H(X) \otimes H(F))$.

If $\lambda$ is the natural lifting function given by path multiplication for the map $p: E X \rightarrow X$, then $\lambda_{*}$ restricts to the map $\lambda_{*}^{\prime}: H_{p}(X) \otimes H_{q}(\Omega X)$ $\rightarrow H_{p+q-1}(\Omega X)$. According to Theorem 2.1, $H_{n}(X)$ can be identified with a direct summand of $H_{n}\left(\bar{B}^{1} A\right) \approx H_{n-1}(\Omega X)$ for $n \geqq 2$. Thus $\lambda_{*}^{\prime}$ can be considered as defining a multiplication between certain elements of $H_{*}(\Omega X)$.

Corollary 2.

(i) $\lambda_{*}^{\prime}$ is Pontryagin multiplication;

(ii) for $n \geqq 1$, the additive structure of $H_{*}(\Omega X)$ is computable from the induction formula

$H_{n}(\Omega X)=\sum_{r+s=n+1 ; s<n} H_{r}(X) \otimes H_{s}(\Omega X)+\sum_{r+s=n ; s<n-1} H_{r}(X) \otimes H_{s}(\Omega X)$.

Proof. Part (i) follows trivially from the definitions; part (ii) follows from Theorem 3.1, since $E X$ is contractible, on computing $H_{n}(\Omega X) \approx H_{n+1}\left(X \times F, x_{0} \times F\right)$ by the Künneth formula and using the relations

$$
H_{0}\left(X, x_{0}\right) \approx H_{1}\left(X, x_{0}\right) \approx 0, \quad H_{i}(X) \approx H_{i}\left(X, x_{0}\right) \quad \text { for } i \geqq 1 .
$$

The formula above was originally shown by G. W. Whitehead to hold if $X$ is a suspension space [3].

Let $E^{r}(\pi)$ denote the Leray-Serre spectral sequence of the fiber map $\pi: T \rightarrow X$. If the differentials $d^{r}$ are all trivial for $r<k, E_{p, q}^{2}(\pi)$ is canonically isomorphic to $E_{p, q}^{k}(\pi)$. Denote by $\sigma: H_{p}(X) \otimes H_{q}(F)$ $\rightarrow E_{p, q}^{k}(\pi)$ the canonical monomorphism of $H_{p}(X) \otimes H_{q}(F)$ into $E_{p, q}^{2}(\pi)$ followed by this isomorphism. Also let $\tau: H_{n}(F) \rightarrow E_{0, n}^{k}(\pi)$ be the canonical epimorphism.

THEOREM 3.2.

(i) $d^{r}: E_{p, q}^{r}(\pi) \rightarrow E_{p-r, q+r-1}^{r}(\pi)$ is zero if $p \neq r$;

(ii) there is a commutative diagram for $p \geqq 2$,

$$
\begin{gathered}
H_{p}(X) \otimes H_{q}(F) \stackrel{-\lambda_{*}^{\prime}}{\longrightarrow} H_{p+q-1}(F) \\
\downarrow \sigma \\
E_{p, q}^{p}(\pi) \stackrel{d^{p}}{\longrightarrow} E_{0, p+q-1}^{p} \quad \downarrow \tau
\end{gathered}
$$


REMARK 1. The exact sequence of Theorem 3.1 exists even if $(F, T, X, \pi, \lambda)$ is not a weakly transitive fiber space, for Brown [2] has shown that every fiber space is fiber homotopically equivalent to a weakly transitive one. Applying Theorem 3.1 to this latter fiber space provides the sequence. Similarly, part (i) of Theorem 3.2 is valid for any fiber space.

Remark 2. If $H(X)$ is free, Corollary 2 says that the Pontryagin algebra $H_{*}(\Omega X)$ is the tensor algebra of $H(X)$ after shifting down by one unit the degrees of elements of $H(X)$. This also follows from a theorem of Bott-Samelson [1], since it is shown in [5] that if cat $(X)$ $\leqq 2$, the homology suspension is an epimorphism and thus all elements of $H(X)$ are transgressive.

4. The general case. If $A \supseteq B$, let $[A, B]^{p}$ denote the $p$-fold cartesian power of the pair.

TheOREM 4.1. Let $(F, T, X, \pi, \lambda)$ be a weakly transitive fiber space with $\pi_{1}(X)=0$ and $\operatorname{cat}(X) \leqq k$. Then there exists a spectral sequence $\dot{E}^{r}$ such that

(i) $\dot{E}^{\infty}$ is the graded group associated with $H(T)$ under a suitable filtration;

(ii) $\dot{E}_{p, q}^{\mathrm{l}}=H_{q}\left([\Omega X, b]^{p} \times F\right)$, where $b$ is the base point of $\Omega X$;

(iii) $d^{r}=0$ for $r \geqq k$;

(iv) $\dot{E}_{p, q}^{\infty}=0$ for $p \geqq k$.

Let $\bar{B} A \tilde{\otimes} C(F)$ (see $\S 1$ ) be filtered by the $\dot{D}_{n}=\sum_{i=0}^{i=n} \bar{B}^{i} A \tilde{\otimes} C(F)$. The $\dot{D}_{n}$ are subcomplexes of the chain complex $\bar{B} A \widetilde{\otimes} C(F)$, and give rise to the spectral sequence $\dot{E}^{r}$ in the usual way. The proof of Theorem 4.1 is now almost word for word a repetition of the proofs of Theorems 2.1,2.2, and 3.1 of [5]. As the proofs of these theorems are long and are given in detail in [5], we will omit a repetition of these arguments.

5. Proof of the theorems. Let $(F, T, X, \pi, \lambda)$ be a weakly transitive fiber space such that $\pi_{1}(X)=0$. Let $A=C(\Omega X)$, and let $\bar{B} A \otimes C(F)$ denote the usual tensor product of chain complexes. $C(F)$ will be identified with the subcomplex $\bar{B}_{0} A \otimes C(F)$. We will consider the following four chain complexes, filtrations, and spectral sequences:

1. $\bar{B} A \widetilde{\otimes} C(F)$ with the filtration $\widetilde{D}_{n}$ and spectral sequence $\tilde{E}^{r}$ defined in $\$ 1$;

2. $\bar{B} A \otimes C(F)$ with the corresponding filtration

$$
D_{n}=\sum_{k=0}^{k=n} \bar{B}_{k} A \otimes C(F)
$$

and spectral sequence $E^{r}$; 
3. $\bar{B} A \tilde{\otimes} C(F) / C(F)$ with the filtration $\widetilde{R}_{n}=\widetilde{D}_{n} / \widetilde{D}_{0}$ and spectral sequence $\tilde{E}^{r}(R)$;

4. $\bar{B} A \otimes C(F) / C(F)$ with the filtration $R_{n}=D_{n} / D_{0}$ and spectral sequence $E^{r}(R)$.

Note that $E^{r}$ and $E^{r}(R)$ are trivial spectral sequences.

The canonical projections $\tilde{\eta}: \bar{B} A \tilde{\otimes} C(F) \rightarrow \bar{B} A \tilde{\otimes} C(F) / C(F)$ and $\eta: \bar{B} A \otimes C(F) \rightarrow \bar{B} A \otimes C(F) / C(F)$ are filtration preserving chain maps and induce maps $\tilde{\eta}_{r}$ and $\eta_{r}$ of the corresponding spectral sequences.

LEMMA 5.1. For $1 \leqq r \leqq p, \eta_{r}: E_{p, q}^{r} \approx E_{p, q}^{r}(R)$ and $\tilde{\eta}_{r}: \tilde{E}_{p, q}^{r} \approx \tilde{E}_{p, q}^{r}(R)$.

Proof. Consider the commutative diagram

$$
\begin{gathered}
D_{p} / D_{p-r} \stackrel{i}{\rightarrow} D_{p+r-1} / D_{p-1} \\
\quad \downarrow \eta^{\prime} \quad \stackrel{j}{ } \quad \downarrow \eta^{\prime \prime} \\
R_{p} / R_{p-r} \stackrel{j}{\rightarrow} R_{p+r-1} / R_{p-1}
\end{gathered}
$$

where the vertical maps are induced by $\eta$ and $i$ and $j$ are inclusions. For $p-r \geqq 0$ and $p-1 \geqq 0$, that is for $1 \leqq r \leqq p$, the vertical maps are isomorphisms of chain complexes. In the induced homology diagram

$$
\begin{array}{cc}
H_{p+q}\left(D_{p} / D_{p-r}\right) & \stackrel{i_{*}}{\rightarrow} H_{p+q}\left(D_{p+r-1} / D_{p-1}\right) \\
\quad \downarrow \eta_{*}^{\prime} & \quad \downarrow \eta_{*}^{\prime \prime} \\
H_{p+q}\left(R_{p} / R_{p-r}\right) & \stackrel{j *}{\rightarrow} H_{p+q}\left(R_{p+r-1} / R_{p-1}\right)
\end{array}
$$

since $\eta_{*}^{\prime}$ and $\eta_{*}^{\prime \prime}$ are isomorphisms, $\eta_{*}^{\prime \prime}$ maps the image of $i_{*}$ isomorphically onto the image of $j_{*}$. But image $i_{*}=E_{p, q}^{r}$, image $j_{*}$ $=E_{p, q}^{r}(R)$, and $\eta_{*}^{\prime \prime} \mid E_{p, q}^{r}=\eta_{r}$. The result for $\tilde{\eta}$ follows by putting tildes over everything in sight.

Now consider the chain map $j \theta: \bar{B} A \rightarrow \bar{B} A$ of Theorem 2.1. Let $i: C(F) \rightarrow C(F)$ be the identity map. Then the map of graded groups

$$
j \theta \otimes i: \bar{B} A \otimes C(F) \rightarrow \bar{B} A \widetilde{\otimes} C(F)
$$

induces a map of graded groups

$$
\psi: \bar{B} A \otimes C(F) / C(F) \rightarrow \bar{B} A \widetilde{\otimes} C(F) / C(F) .
$$

LEMMA 5.2. $\psi$ is a chain equivalence if $\operatorname{cat}(X) \leqq 2$.

Proof. If $\left[a_{1}, \cdots, a_{n}\right] \in \bar{B}_{p} A$, we can write $j \theta\left[a_{1}, \cdots, a_{n}\right]$ $=\sum_{j}\left[b_{j}\right]$ for some $b_{j} \in A$. Using this and the explicit form of the boundary operators $\tilde{d}$ in $\bar{B} A \widetilde{\otimes} C(F)$ and $d$ in $\bar{B} A \otimes C(F)$, an elementary calculation shows that

$$
[\tilde{d}(j \theta \otimes i)-(j \theta \otimes i) d]\left[a_{1}, \cdots, a_{n}\right] \otimes c=-\sum_{j}[] \otimes b_{j} \cdot c \in C(F) .
$$


Hence $\tilde{d} \psi=\psi d$ and $\psi$ is a chain map.

Since $\psi$ is clearly filtration preserving, it induces maps $\psi_{r}: E^{r}(R)$ $\rightarrow \tilde{E}^{r}(R)$. Furthermore, since $\pi_{1}(X)=0$, it is shown in [2] that there are natural isomorphisms

$$
\lambda: \bar{B}_{p} A \otimes H_{q}(F) \approx E_{p, q}^{1} \text { and } \tilde{\lambda}: \bar{B}_{p} A \otimes H_{q}(F) \approx \tilde{E}_{p, q}^{1},
$$

where in each case, $\bar{B} A \otimes H(F)$ has the boundary operator $\bar{d} \otimes 1$. A routine calculation shows that the diagram

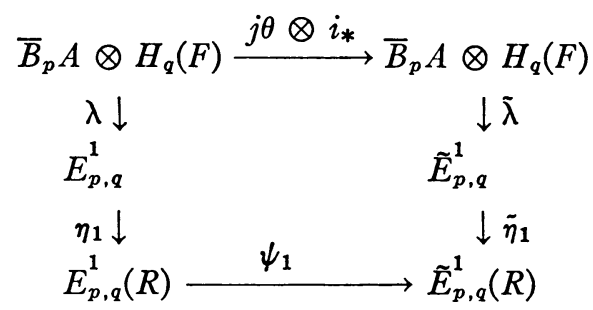

is commutative. By Lemma 5.1, $\eta_{1}$ and $\tilde{\eta}_{1}$ are group isomorphisms for $p \geqq 1$, and so are isomorphisms of complexes for $p \geqq 2$. Since $j_{*} \theta_{*}$ is the identity isomorphism by Theorem 2.1 , it follows that $\psi_{2}: E_{p, q}^{2}(R)$ $\approx \tilde{E}_{p, q}^{2}(R)$ for all $p \geqq 2$. Since $E_{p, q}^{2}(R) \approx \tilde{E}_{p, q}^{2}(R) \approx 0$ for $p \leqq 1, \psi_{2}$ is an isomorphism. By a standard argument, so is $\psi_{*}$. As all the chain complexes considered are free, $\psi$ is a chain equivalence.

Proof of Theorem 3.1. According to Theorem 1.1 we may replace the exact homology sequence of $(T, F)$ by that of $(\bar{B} A \tilde{\otimes} C(F), C(F))$. By Lemma 5.2, the relative group in this sequence is $H(\bar{B} A \otimes C(F)$, $C(F)$ ), which, by the Künneth theorem, is $H\left(X \times F, x_{0} \times F\right)$. Hence the sequence of Theorem 3.1 exists and $f$ is induced by inclusion.

Let $\bar{\mu} \in H_{n}(X \times F)$ be represented by the cycle $\mu=\sum_{i} b_{i} \otimes c_{i}$ of $\bar{B} A \otimes C(F)$. Then $\theta\left(b_{i}\right)=\sum_{j}\left[b_{i j}\right]+r_{i}[]$ for some $b_{i j} \in \bar{A}$ and $r_{i} \in Z$. By definition, $\lambda_{*}(\bar{\mu})$ is represented by

$\bar{\lambda}(S \theta \otimes 1)(\mu)=\bar{\lambda}(S \otimes 1)\left[\sum_{i j}\left[b_{i j}\right] \otimes c_{i}+\sum_{i} r_{i}[] \otimes c_{i}\right]=\sum_{i j} b_{i j} \cdot c_{i}$, while $g i_{*}(\bar{\mu})$ is represented by

$$
\tilde{d} \psi i(\mu)=\tilde{d}(j \theta \otimes 1)(\mu)=(j \theta \otimes 1) d(\mu)-\sum_{i j}[] \otimes b_{i j} \cdot c_{i} .
$$

Since $d(\mu)=0, g i_{*}(\bar{\mu})=-\lambda_{*}(\bar{\mu})$, and the theorem is proven.

Proof of Theorem 3.2. By Theorem 1.1, we may replace $E^{r}(\pi)$ by $\tilde{E}^{r}$. In proving Lemma 5.2 we have shown that $\psi_{r}: E^{r}(R) \rightarrow \tilde{E}^{r}(R)$ is an isomorphism for $r \geqq 2$; thus $\widetilde{E}^{r}(R)$ is a trivial spectral sequence. By Lemma 5.1, $\tilde{\eta}_{r}: \tilde{E}_{p, q}^{r} \rightarrow \tilde{E}_{p, q}^{r}(R)$ is an isomorphism for $p \geqq r$, which implies $\tilde{d}^{r}: \tilde{E}_{p, q}^{r} \rightarrow \tilde{E}_{p-r, q+r-1}^{r}$ is trivial for $p>r . \tilde{d}^{r}=0$ for $p<r$ trivially, so $\tilde{d}^{r}=0$ for $r \neq p$. 
Letting a generator $\mu \in H_{p}(X) \otimes H_{q}(F)$ be represented by $b \otimes c$, where $b$ and $c$ are cycles, we have, as in the proof of Theorem 3.1, $\tau \lambda_{*}(\mu)$ represented by $\sum_{j} b_{j} \cdot c$. On the other hand, $j \theta(b)=\sum_{j}\left[b_{j}\right]$ is homologous to $b$ since $j_{*} \theta_{*}$ is the identity isomorphism, according to Theorem 2.1. Hence $\mu$ is also represented by $\sum_{j}\left[b_{j}\right] \otimes c$. As $\tilde{d} \sum_{j}\left[b_{j}\right] \otimes c=-\sum_{j}[] \otimes b_{j} \cdot c \in \tilde{D}_{0}, \quad \sigma(\mu)$ is also represented by $\sum_{j}\left[b_{j}\right] \otimes c$, while $\tilde{d}^{p} \sigma(\mu)$ has $-\sum_{j}[] \otimes b_{j} \cdot c$ as representative. Thus $\tilde{d}^{p} \sigma=-\tau \lambda_{*}$.

\section{REFERENCES}

1. R. Bott and H. Samelson, On the Pontryagin product in spaces of paths, Comment. Math. Helv. 27 (1953), 320-337.

2. E. H. Brown, Twisted tensor products. I, Ann. of Math. (2) 69 (1959), 223-246.

3. H. Cartan, Algèbres d'Eilenberg-MacLane et homotopie, Séminaire H. Cartan, Ecole Normale Supérieure, Paris, 1954-1955.

4. S. Eilenberg and S. MacLane, On the groups $H(\pi, n)$. I, Ann. of Math. (2) 58 (1953), 55-106.

5. M. Ginsburg, On the Lusternik-Schnirelmann category, Ann. of Math. (2) 77 (1963), 538-551.

6. - Categorical invariants and fiber spaces, Colloq. on Algebraic Topology, Aarhus Universitet, Aarhus, Denmark, 1962.

7. D. Puppe, Homotopiemengen und ihre induzierten Abbildungen. I, Math. Z. 69 (1958), 299-344.

8. J.-P. Serre, Homologie singulière des espaces fibrés. Applications, Ann. of Math. (2) 54 (1951), 425-505.

University of Chicago 